\title{
HOLOMORPHIC HARMONIC MORPHISMS FROM FOUR-DIMENSIONAL NON-EINSTEIN MANIFOLDS
}

\author{
SIGMUNDUR GUDMUNDSSON
}

version 1.008 - 21 May 2014

\begin{abstract}
We construct 4-dimensional Riemannian Lie groups carrying leftinvariant conformal foliations with minimal leaves of codimension 2. We show that these foliations are holomorphic with respect to an (integrable) Hermitian structure which is not Kähler. We then prove that the Riemannian Lie groups constructed are not Einstein manifolds. This answers an important open question in the theory of complex-valued harmonic morphisms from Riemannian 4-manifolds.
\end{abstract}

\section{INTRODUCTION}

More than twenty years ago, J. C. Wood proved, in [7, that any submersive harmonic morphism from an orientable 4-dimensional Einstein manifold $M$ to a Riemann surface, or a conformal foliation of $M$ by minimal surfaces, determines an (integrable) Hermitian structure with respect to which it is holomorphic. Ever since it has been an open question whether holomorphicity in the above situation forces the 4-manifold $M$ to be Einstein.

In this paper we construct two 3-dimensional families of 4-dimensional Riemannian Lie groups carrying left-invariant conformal foliations with minimal leaves of codimension 2. We show that these foliations are holomorphic with respect to an (integrable) Hermitian structure which is not Kähler. We then prove that the Riemannian Lie groups constructed are not Einstein manifolds. This gives a definite answer to the above mentioned open question.

For the general theory of harmonic morphisms between Riemannian manifolds we refer to the excellent book [2] and the regularly updated on-line bibliography [4].

\section{HARMONIC MORPHISMS AND MINIMAL CONFORMAL FOLIATIONS}

Let $M$ and $N$ be two manifolds of dimensions $m$ and $n$, respectively. A Riemannian metric $g$ on $M$ gives rise to the notion of a Laplacian on $(M, g)$ and real-valued harmonic functions $f:(M, g) \rightarrow \mathbb{R}$. This can be generalized to the concept of harmonic maps $\phi:(M, g) \rightarrow(N, h)$ between Riemannian

2010 Mathematics Subject Classification. 58E20, 53C43, 53C12.

Key words and phrases. harmonic morphisms, holomorphic, Einstein manifolds. 
manifolds, which are solutions to a semi-linear system of partial differential equations, see [2].

Definition 2.1. A map $\phi:(M, g) \rightarrow(N, h)$ between Riemannian manifolds is called a harmonic morphism if, for any harmonic function $f: U \rightarrow \mathbb{R}$ defined on an open subset $U$ of $N$ with $\phi^{-1}(U)$ non-empty, $f \circ \phi: \phi^{-1}(U) \rightarrow$ $\mathbb{R}$ is a harmonic function.

The following characterization of harmonic morphisms between Riemannian manifolds is due to Fuglede and T. Ishihara. For the definition of horizontal (weak) conformality we refer to 2 .

Theorem 2.2. [3, 6] A map $\phi:(M, g) \rightarrow(N, h)$ between Riemannian manifolds is a harmonic morphism if and only if it is a horizontally (weakly) conformal harmonic map.

Let $(M, g)$ be a Riemannian manifold, $\mathcal{V}$ be an involutive distribution on $M$ and denote by $\mathcal{H}$ its orthogonal complement distribution on $M$. As customary, we also use $\mathcal{V}$ and $\mathcal{H}$ to denote the orthogonal projections onto the corresponding subbundles of $T M$ and denote by $\mathcal{F}$ the foliation tangent to $\mathcal{V}$. The second fundamental form for $\mathcal{V}$ is given by

$$
B^{\mathcal{V}}(U, V)=\frac{1}{2} \mathcal{H}\left(\nabla_{U} V+\nabla_{V} U\right) \quad(U, V \in \mathcal{V}),
$$

while the second fundamental form for $\mathcal{H}$ satisfies

$$
B^{\mathcal{H}}(X, Y)=\frac{1}{2} \mathcal{V}\left(\nabla_{X} Y+\nabla_{Y} X\right) \quad(X, Y \in \mathcal{H}) .
$$

The foliation $\mathcal{F}$ tangent to $\mathcal{V}$ is said to be conformal if there is a vector field $V \in \mathcal{V}$ such that

$$
B^{\mathcal{H}}=g \otimes V,
$$

and $\mathcal{F}$ is said to be Riemannian if $V=0$. Furthermore, $\mathcal{F}$ is said to be minimal if trace $B^{\mathcal{V}}=0$ and totally geodesic if $B^{\mathcal{V}}=0$. This is equivalent to the leaves of $\mathcal{F}$ being minimal and totally geodesic submanifolds of $M$, respectively.

It is easy to see that the fibres of a horizontally conformal map (resp. Riemannian submersion) give rise to a conformal foliation (resp. Riemannian foliation). Conversely, the leaves of any conformal foliation (resp. Riemannian foliation) are locally the fibres of a horizontally conformal map (resp. Riemannian submersion), see [2].

The next result of Baird and Eells gives the theory of harmonic morphisms, with values in a surface, a strong geometric flavour.

Theorem 2.3. [1] Let $\phi:\left(M^{m}, g\right) \rightarrow\left(N^{2}, h\right)$ be a horizontally conformal submersion from a Riemannian manifold to a surface. Then $\phi$ is harmonic if and only if $\phi$ has minimal fibres. 


\section{4-DIMENSIONAL LIE GROUPS}

Let $G$ be a 4-dimensional Lie group equipped with a left-invariant Riemannian metric. Let $\mathfrak{g}$ be the Lie algebra of $G$ and $\{X, Y, Z, W\}$ be an orthonormal basis for $\mathfrak{g}$. Let $Z, W \in \mathfrak{g}$ generate a 2-dimensional left-invariant and integrable distribution $\mathcal{V}$ on $G$ which is conformal and with minimal leaves. We denote by $\mathcal{H}$ the horizontal distribution, orthogonal to $\mathcal{V}$, generated by $X, Y \in \mathfrak{g}$. Then it is easily seen that the basis $\{X, Y, Z, W\}$ can be chosen so that the Lie bracket relations for $\mathfrak{g}$ are of the form

$$
\begin{aligned}
{[W, Z] } & =\lambda W \\
{[Z, X] } & =\alpha X+\beta Y+z_{1} Z+w_{1} W, \\
{[Z, Y] } & =-\beta X+\alpha Y+z_{2} Z+w_{2} W, \\
{[W, X] } & =a X+b Y+z_{3} Z-z_{1} W, \\
{[W, Y] } & =-b X+a Y+z_{4} Z-z_{2} W, \\
{[Y, X] } & =r X+\theta_{1} Z+\theta_{2} W
\end{aligned}
$$

with real structure constants. For later reference we state the following easy result describing the geometry of the situation.

Proposition 3.1. Let $G$ be a 4-dimensional Lie group and $\{X, Y, Z, W\}$ be an orthonormal basis for its Lie algebra as above. Then

(i) $\mathcal{F}$ is totally geodesic if and only if $z_{1}=z_{2}=z_{3}+w_{1}=z_{4}+w_{2}=0$,

(ii) $\mathcal{F}$ is Riemannian if and only if $\alpha=a=0$, and

(iii) $\mathcal{H}$ is integrable if and only if $\theta_{1}=\theta_{2}=0$.

On the Riemannian Lie group $G$ there exist, up to sign, exactly two invariant almost Hermitian structure $J_{1}$ and $J_{2}$ which are adapted to the orthogonal decomposition $\mathfrak{g}=\mathcal{V} \oplus \mathcal{H}$ of the Lie algebra $\mathfrak{g}$. They are determined by

$$
\begin{aligned}
& J_{1} X=Y, J_{1} Y=-X, J_{1} Z=W, J_{1} W=-Z, \\
& J_{2} X=Y, J_{2} Y=-X, J_{2} W=Z, J_{2} Z=-W .
\end{aligned}
$$

An elementary calculation involving the Nijenhuis tensor shows that $J_{1}$ is integrable if and only if

$$
2 z_{1}-z_{4}-w_{2}=2 z_{2}+z_{3}+w_{1}=0 .
$$

In this case the Lie bracket relations for $\mathfrak{g}$ take the form

$$
\begin{aligned}
{[W, Z] } & =\lambda W, \\
{[Z, X] } & =\alpha X+\beta Y+z_{1} Z-\left(2 z_{2}+z_{3}\right) W, \\
{[Z, Y] } & =-\beta X+\alpha Y+z_{2} Z+\left(2 z_{1}-z_{4}\right) W, \\
{[W, X] } & =a X+b Y+z_{3} Z-z_{1} W, \\
{[W, Y] } & =-b X+a Y+z_{4} Z-z_{2} W, \\
{[Y, X] } & =r X+\theta_{1} Z+\theta_{2} W .
\end{aligned}
$$


Example 3.2. With the non-vanishing coefficients $z_{2}, \theta_{1}, \theta_{2}$ we obtain the following 3-dimensional family of Lie algebras

$$
\begin{aligned}
{[Z, X] } & =-2 z_{2} W, \\
{[Z, Y] } & =z_{2} Z, \\
{[W, Y] } & =-z_{2} W, \\
{[Y, X] } & =2 z_{2} X+\theta_{1} Z+\theta_{2} W .
\end{aligned}
$$

These are the special cases $\mathfrak{g}_{7}\left(z_{2},-2 z_{2}, 0, \theta_{1}, \theta_{2}\right)$ of Example 5.3 in [5]. It should be noted that the horizontal distribution $\mathcal{H}$ is not integrable and the leaves of the vertical foliation $\mathcal{V}$ are not totally geodesic.

We will show that none of the corresponding Riemannian Lie groups are Einstein manifolds. A standard calculation involving the Koszul fomula

$$
2\left\langle\nabla_{X} Y, Z\right\rangle=\langle[Z, X], Y\rangle+\langle[Z, Y], X\rangle+\langle Z,[X, Y]\rangle
$$

shows that the Levi-Civita connection satisfies the following relations

$$
\begin{gathered}
\nabla_{X} X=2 z_{2} Y, \quad \nabla_{X} Y=-2 z_{2} X-\frac{1}{2} \theta_{1} Z-\frac{1}{2} \theta_{2} W \\
\nabla_{X} Z=\frac{1}{2} \theta_{1} Y+z_{2} W, \quad \nabla_{X} W=\frac{1}{2} \theta_{2} Y-z_{2} Z, \\
\nabla_{Y} X=\frac{1}{2} \theta_{1} Z+\frac{1}{2} \theta_{2} W, \quad \nabla_{Y} Y=0, \\
\nabla_{Y} Z=-\frac{1}{2} \theta_{1} X, \quad \nabla_{Y} W=-\frac{1}{2} \theta_{2} X \\
\nabla_{Z} X=\frac{1}{2} \theta_{1} Y-z_{2} W, \quad \nabla_{Z} Y=-\frac{1}{2} \theta_{1} X+z_{2} Z, \\
\nabla_{Z} Z=-z_{2} Y, \quad \nabla_{Z} W=z_{2} X \\
\nabla_{W} X=\frac{1}{2} \theta_{2} Y-z_{2} Z, \quad \nabla_{W} Y=-\frac{1}{2} \theta_{2} X-z_{2} W \\
\nabla_{W} Z=z_{2} X, \quad \nabla_{W} W=z_{2} Y .
\end{gathered}
$$

This means that the Hermitian structure $J_{1}$ is not Kähler, since

$$
\left(\nabla_{X} J_{1}\right)(X)=-\frac{1}{2}\left(\theta_{1} Z+\theta_{2} W\right) .
$$

Employing the definition for the sectional curvature

$$
\langle R(X, Y) Y, X\rangle=\left\langle\nabla_{X} \nabla_{Y} Y-\nabla_{Y} \nabla_{X} Y-\nabla_{[X, Y]} Y, X\right\rangle
$$

we then obtain the following useful equalities

$$
\begin{gathered}
\langle R(X, Y) Y, X\rangle=-\frac{3}{4}\left(\theta_{1}^{2}+\theta_{2}^{2}\right)-4 z_{2}^{2}, \quad\langle R(X, Z) Z, X\rangle=\frac{1}{4} \theta_{1}^{2}-z_{2}^{2}, \\
\langle R(X, W) W, X\rangle=\frac{1}{4} \theta_{2}^{2}-z_{2}^{2}, \quad\langle R(Y, Z) Z, Y\rangle=\frac{1}{4} \theta_{1}^{2}-z_{2}^{2}, \\
\langle R(Y, W) W, Y\rangle=\frac{1}{4} \theta_{2}^{2}-z_{2}^{2}, \quad\langle R(Z, W) W, Z\rangle=2 z_{2}^{2} . \\
4
\end{gathered}
$$


One immediate consequence is that

$$
\operatorname{Ric}(X, X)=-\frac{1}{2}\left(\theta_{1}^{2}+\theta_{2}^{2}\right)-6 z_{2}^{2}, \quad \operatorname{Ric}(Z, Z)=\frac{1}{2} \theta_{1}^{2} .
$$

showing that none of these Riemannian Lie groups is an Einstein manifold.

\section{The structures $J_{1}$ And $J_{2}$ ARe both integrable}

It is easily seen that $J_{1}$ and $J_{2}$ are both integrable if and only if

$$
\begin{aligned}
& 2 z_{1}-z_{4}-w_{2}=2 z_{2}+z_{3}+w_{1}=0, \\
& 2 z_{1}+z_{4}+w_{2}=2 z_{2}-z_{3}-w_{1}=0 .
\end{aligned}
$$

As a direct consequence, we see that in this case the foliation $\mathcal{F}$ is totally geodesic i.e.

$$
z_{1}=z_{2}=z_{3}+w_{1}=z_{4}+w_{2}=0 .
$$

In this situation the Lie bracket relations take the following form

$$
\begin{aligned}
{[W, Z] } & =\lambda W, \\
{[Z, X] } & =\alpha X+\beta Y-z_{3} W, \\
{[Z, Y] } & =-\beta X+\alpha Y-z_{4} W, \\
{[W, X] } & =a X+b Y+z_{3} Z, \\
{[W, Y] } & =-b X+a Y+z_{4} Z, \\
{[Y, X] } & =r X+\theta_{1} Z+\theta_{2} W .
\end{aligned}
$$

Example 4.1. For the non-vanishing coefficients $\alpha, \beta, \theta_{2}$ we have the following 3-dimensional family of Lie algebras

$$
\begin{aligned}
& {[W, Z]=-2 \alpha W,} \\
& {[Z, X]=\alpha X+\beta Y,} \\
& {[Z, Y]=-\beta X+\alpha Y,} \\
& {[Y, X]=\theta_{2} W .}
\end{aligned}
$$

They are the special cases $\mathfrak{g}_{3}\left(\alpha, \beta, 0,0, \theta_{2}\right)$ of Example 4.3 in [5]. For the corresponding Riemannian Lie groups the Levi-Civita connection is given by

$$
\begin{gathered}
\nabla_{X} X=\alpha Z, \quad \nabla_{X} Y=-\frac{1}{2} \theta_{2} W, \quad \nabla_{X} Z=-\alpha X, \quad \nabla_{X} W=\frac{1}{2} \theta_{2} Y, \\
\nabla_{Y} X=\frac{1}{2} \theta_{2} W, \quad \nabla_{Y} Y=\alpha Z, \quad \nabla_{Y} Z=-\alpha Y, \quad \nabla_{Y} W=-\frac{1}{2} \theta_{2} X, \\
\nabla_{Z} X=\beta Y, \quad \nabla_{Z} Y=-\beta X, \quad \nabla_{Z} Z=0, \quad \nabla_{Z} W=0, \\
\nabla_{W^{X}}=\frac{1}{2} \theta_{2} Y, \quad \nabla_{W} Y=-\frac{1}{2} \theta_{2} X, \quad \nabla_{W} Z=-2 \alpha W, \quad \nabla_{W} W=2 \alpha Z .
\end{gathered}
$$

Using these identities it is easily seen that

i) the Hermitian structure $J_{1}$ is Kähler if and only if $\theta_{2}=-2 \alpha$,

ii) the Hermitian structure $J_{2}$ is Kähler if and only if $\theta_{2}=2 \alpha$. 
This means that in all the cases that we are considering at least one of the Hermitian structures $J_{1}$ or $J_{2}$ is not Kähler. For the sectional curvatures have

$$
\begin{gathered}
\langle R(X, Y) Y, X\rangle=-\alpha^{2}-\frac{3}{4} \theta_{2}^{2}, \quad\langle R(X, Z) Z, X\rangle=-\alpha^{2}, \\
\langle R(X, W) W, X\rangle=\frac{1}{4} \theta_{2}^{2}-2 \alpha^{2}, \quad\langle R(Y, Z) Z, Y\rangle=-\alpha^{2}, \\
\langle R(Y, W) W, Y\rangle=\frac{1}{4} \theta_{2}^{2}-2 \alpha^{2}, \quad\langle R(Z, W) W, Z\rangle=-4 \alpha^{2} .
\end{gathered}
$$

Finally, we yield

$$
\operatorname{Ric}(X, X)=-\frac{1}{2} \theta_{2}^{2}-4 \alpha^{2}, \quad \operatorname{Ric}(Z, Z)=-6 \alpha^{2}, \quad \operatorname{Ric}(W, W)=\frac{1}{2} \theta_{2}^{2}-8 \alpha^{2}
$$

telling us that if $4 \alpha^{2} \neq \theta_{2}^{2}$ then our Riemannian Lie group is not an Einstein manifold.

\section{ACKNOWLEDGEMEnTS}

The author is grateful to John $\mathbb{C}$. Wood for useful discussions on this work at the Differential Geometry Workshop held at Lund in May 2014.

\section{REFERENCES}

[1] P. Baird and J. Eells, A conservation law for harmonic maps, Geometry Symposium Utrecht 1980, Lecture Notes in Mathematics 894, 1-25, Springer (1981).

[2] P. Baird and J. C. Wood, Harmonic morphisms between Riemannian manifolds, London Math. Soc. Monogr. No. 29, Oxford Univ. Press (2003).

[3] B. Fuglede, Harmonic morphisms between Riemannian manifolds, Ann. Inst. Fourier 28 (1978), 107-144.

[4] S. Gudmundsson, The Bibliography of Harmonic Morphisms, http://www.matematik.lu.se/ matematiklu/personal/sigma/harmonic/bibliography.html

[5] S. Gudmundsson, M. Svensson, Harmonic morphisms from four-dimensional Lie groups, J. Geom. Phys. 83 (2014), 1-11.

[6] T. Ishihara, A mapping of Riemannian manifolds which preserves harmonic functions, J. Math. Soc. Japan 7 (1979), 345-370.

[7] J. C. Wood, Harmonic morphisms and Hermitian structures on Einstein 4-manifolds, Internat. J. Math. 3 (1992), 415439.

Department of Mathematics, Faculty of Science, Lund University, Box 118, S-221 00 Lund, SWEDEN

E-mail address: Sigmundur.Gudmundsson@math.lu.se 